\title{
CONSTRUCCIÓN Y APLICACIÓN DE UN ÍNDICE DE SATISFACCIÓN DEL USUARIO (I.S.U) ADECUADA A LA DEMANDA DEL SECTOR ALOJAMIENTO DE LA CIUDAD DE NEUQUÉN
}

\author{
Autores \\ Lorenna Lombardo \\ María Alejandra Gazzera \\ Fabiana Quadrini \\ Marcela Molina \\ Gabriela Marenzana \\ Mónica Vogel \\ Docentes \\ Facultad de Turismo \\ Universidad Nacional del Comahue \\ Neuquén, Argentina
}

\section{RESUMEN:}

El Turismo en la ciudad de Neuquén está en franco proceso de crecimiento, y de pasar a ser considerada un centro de escala, desde el plano Municipal y Provincial es evidente el interés político y estratégico de que se convierta en un centro de estadía a partir de la revalorización y aumento de la oferta de atractivos culturales, entretenimiento, etc. de la ciudad, para lo cual muchas Empresas de Viajes y Turismo observando las tendencias propias del mercado de viajes reconvirtieron su actividad emisivas a receptivas o mejor aún se incorporaron nuevas Agencias de Viajes como receptivas exclusivamente en la ciudad de Neuquén.

En ésta línea, la competencia es importante y los cambios de tendencia en el sector se suceden vertiginosamente. El consumidor turístico es cada vez más exigente, exige calidad en los servicios, inmediatez en las respuestas y busca experiencias que le hagan sentirse diferente y especial. Ante este panorama, los dueños/gerentes de alojamientos se dan cuenta de lo beneficioso que es trabajar por la satisfacción de sus clientes, (aunque no esté instalada la cultura de medir desempeño) no solo para retenerlos, también para incrementar su nivel de consumo y para atraer otros nuevos mediante su recomendación, lo cual el tipo de proyecto presentado permite dar muestras de la viabilidad del mismo.

Por ello es que se decide proyectar nuevas alternativas de herramientas para la gestión de la calidad como es el Índice de Satisfacción del Usuario del sector alojamiento de la ciudad de Neuquén, como alternativa de diagnóstico y soluciones al problema de la falta de medición de la calidad.

Palabras Claves: Medición, construcción, índice de satisfacción del usuario (I.S.U), demanda, sector alojamiento, Neuquén. 


\title{
CONSTRUCTION AND IMPLEMENTATION OF AN INDEX OF USER SATISFACTION (ISU) TO KEEP PACE WITH DEMAND ACCOMMODATION IN THE FIELD OF THE CITY OF NEUQUÉN
}

\author{
Authors \\ Lorenna Lombardo \\ María Alejandra Gazzera \\ Fabiana Quadrini \\ Marcela Molina \\ Gabriela Marenzana \\ Mónica Vogel \\ Teachers \\ School of Tourism \\ National University of Comahue \\ Neuquén, Argentina
}

\section{ABSTRACT}

Tourism in the city of Neuquén is in frank process of growth, and to being considered as a scale center, from the Municipal and Provincial point of view, it is evident the political and strategic interest that it should become a stay center from the revaluation and increase of the supply of cultural, entertainment attractions, etc. of the city, this is the reason why many Travel Agencies and Tourist companies, observing the tendencies of the travel market reconverted their activity from missive to receptive, or even better, new Travel Agencies exclusively as receptive were incorporated in the city of Neuquén.

On this subject, competition is important and the changes of tendency in the field occur vertiginously. The tourist consumer is more and more demanding, they demand quality in services, quick answers and looks for experiences that make them feel different and special. Having this situation in mind, lodgings owners/managers realize the benefits of working for the satisfaction of their clients, (although the culture of measuring performance is not installed) not only to retain them, but also to increase their level of consumption and to attract new ones by means of their recommendation. This type of research allows giving samples of its viability.

For that reason it is decided to project new alternatives of tools for quality management such as the User Satisfaction Index of lodging facilities in the city of Neuquén, as a diagnosis and solution alternative to the problem of the lack of measurement of quality in service.

Key Words: Measurement, construction, user satisfaction index (I.S.U), lodging, demand, Neuquén. 


\section{MARCO TEORICO}

La calidad no es un atributo ni privilegio de grandes empresas, está referida al gerenciamiento del negocio y abarca todos los aspectos, cualquiera sea su tamaño, inclusive una empresa unipersonal puede estar involucrada en un programa de calidad. Sin embargo si bien conceptos como Calidad y Satisfacción están entrelazados hay diferencias entre estos. Hay quienes piensan que la Satisfacción de los clientes produce la Calidad percibida en los servicios mientras otros piensan que la Calidad lleva a la Satisfacción del cliente. La relación que existe entre estos dos conceptos y la forma en que están relacionados con el comportamiento de compra prácticamente no ha sido explicada (Cronin y Taylor, 1992). Asimismo Cronin y Taylor (1992) argumentan que una explicación probable sería que la satisfacción ayuda a los consumidores a revisar sus percepciones acerca de la calidad de servicios.

La lógica que sustenta esta posición dice que:

1) Las percepciones de los consumidores con respecto a la calidad de servicios de una empresa con la cual no ha tenido experiencias anteriores están fundamentadas en sus expectativas

2) Los encuentros posteriores con la empresa llevan al consumidor por el proceso de "Desmentido" (modelo de las expectativas desmentidas) y de ahí a revisar sus percepciones acerca de la calidad de los servicios

3) Cada encuentro adicional con la empresa llevan al consumidor a revisar y reforzar aún más las percepciones con respecto a los servicios, es decir las percepciones revisadas relativas a la calidad del servicio modifican las interacciones del consumidor para comprar en el futuro de esa empresa. Para ofrecerles a los clientes un conjunto consistente de experiencias satisfactorias que se traduzcan en una elevada calidad, se necesita que toda la organización se concentre en saber qué necesita los clientes, saber las limitaciones de las operaciones con que opera y brindar servicios de acuerdo al diseño.

Si bien existen varias definiciones alternativas de satisfacción/insatisfacción de los clientes (Keith Hunt ,1991), la más popular dice que se trata de una comparación de las expectativas de los consumidores con sus percepciones relacionadas con el encuentro real del servicio.

Muchos trabajos han intentado medir la satisfacción midiendo sólo el componente cognitivo .Maestro et al (2002) citan a Oliver (1980), y recomiendan recabar también el componente afectivo de (Oliver, 1983), Wirtz y Bateson (1999) y Bigné y Andreu (2002).En la actualidad la medición de la satisfacción del cliente constituye un objetivo importantísimo para las empresas a la hora de valorar resultados económicos como los beneficios, las respuestas a las inversiones, etc.pero no debe considerarse una panacea 
Beneficia a una empresa mientras no se convierta en un fin.

La satisfacción del consumidor es una medida adicional relativa a la calidad del producto o servicio y está íntimamente relacionada con el mantenimiento de la fidelidad del cliente dentro del mercado, lo cual provoca probabilidad de recompra y recomendaciones a los demás. Aguirre, Caro y Mariel (2005) citan los índices sobre la satisfacción del consumidor (SC) tanto a nivel nacional como internacional que han sido desarrollaos tanto en Suecia, Noruega, Estados Unidos, Dinamarca, etc. Asimismo citan a Fornell (1992) y Fornell et al (1996) en su modelo de Satisfacción Global del Consumidor (SGC), centrándose en los procesos que rodean la satisfacción del consumidor teniendo encuentra sus antecedentes y sus implicancias o consecuencias. Los antecedentes son: la calidad percibida del servicio, la imagen que los consumidores obtienen de un servicio, la marca o empresa y el valor percibido. Por otro lado las consecuencias son la lealtad, la fidelidad, la probabilidad de recompra y recomendaciones los demás, junto con las quejas, de las que se mide su frecuencia.

Con respecto a la calidad percibida, el grupo de investigación (Tamagni L, Gazzera, Lombardo, Zanfardini, et al,) se encuentra trabajando desde 1998 en la aplicación y adecuación del modelo Servqual (Parasuraman, Berry, Zeimthanl, 1985) aplicadas a distintos tipos de servicios como transporte aéreo, agencias de viajes y hotelería a nivel de la región patagónica. El resultado de estas investigaciones son nuevas propuestas de operacionalización de la calidad percibida generando escala de medición. Estos trabajos servirán de marco de referencia para el presente proyecto.

Por otro lado, las normas IRAM-ISO 9001 establecen un apartado para el seguimiento y medición de la satisfacción del cliente como prioritario a la hora de gestionar el desempeño de la calidad, pero no establece cómo llevarlas a cabo. Lo cual este proyecto permitirá proveer de los lineamientos necesarios.

La calidad en su concepto profesional debe entenderse como tecnología aplicada a la empresa, es decir como el conjunto de herramientas, metodologías y conocimientos que facilitan la mejora continua y sistemática de la eficiencia empresarial incluyendo la propia gestión y los servicios prestados. Para evaluar esto es necesario establecer indicadores numéricos que permitan medir y definir la situación referente a calidad y nivel de satisfacción 


\section{OBJETIVOS \\ Objetivo General:}

Construir y aplicar un Índice de Satisfacción del usuario (I.S.U) adecuada a la demanda del sector Alojamiento de la ciudad de Neuquén que facilite a los dueños/ gerentes de las pequeñas y medianas empresas hoteleras potenciar su negocio mediante la incorporación rápida a su práctica gerencial

\section{Objetivos Específicos}

- Obtener un índice de satisfacción del cliente a nivel individual por empresa y general para el sector hotelero de Neuquén capital

- Desarrollar lineamientos de medición, para que la organización pueda determinar qué, cómo, quién, y cuándo medir las necesidades del sector en función de las necesidades del cliente.

- Capacitar a la organización en cuanto al aprovechamiento, aplicación y uso del I.S.U

\section{METODOLOGIA:}

En la primera etapa del trabajo se utilizó información generada por otros estudios de campo e investigación realizados por integrantes del equipo de trabajo, principalmente para la elaboración del marco teórico-referencial, variables e indicadores de calidad y el armado del índice de satisfacción generado. Para la obtención de información específica con el fin de cumplir los objetivos de la investigación presentada, se utilizaron herramientas "vivenciales" en forma directa.

\section{a).- Encuestas a turistas}

Los hoteles seleccionados para el siguiente trabajo, fueron los establecimientos de cuatro, tres y dos estrellas. Para el caso de los hoteles cuatro estrellas, Neuquén solo cuenta con un establecimiento. Los hoteles tres estrellas son seis en total, los cuales fueron relevados en su totalidad, mientras que para el caso de los hoteles dos estrellas, solo fueron relevados tres.

El número de encuestas sugeridas, se propuso teniendo en cuenta la cantidad de plazas ofrecidas, lo que resulta un total de 30 encuestas para los hoteles 2 y 3 estrellas y 59 para el único caso del hotel cuatro estrellas.

Debido que en algunos casos fue imposible llegar al número de encuestas sugeridas, es que se decide tomar solo 24 encuestas Se realizaron un total de 325 encuestas estructuradas, durante el año 2007 en temporada alta y baja durante los meses de julio, junio, agosto, septiembre y octubre a los efectos de captar tanto al consumidor corporativo como al turista de paso por la ciudad de Neuquén 


\section{b).- Entrevistas en profundidad}

Se realizaron entrevistas en profundidad a los dueños-gerentes representativos de los establecimientos anteriormente señalados con el objetivo de recabar información primaria referente a la oferta hotelera del área de estudio

\section{c).- Realización de talleres}

Una de las metas propuestas en el trabajo, fue la de transmitir a las empresas cómo obtener cada una su propio índice de satisfacción (I.S.U.) A los efectos de cumplir con dicha meta, el equipo propuso el taller como una forma instituyente de Capacitación, para que los integrantes del sector sean los generadores de sus propias soluciones, a través del conocimiento, interpretación, comprensión y transformación de su realidad, entendiendo por capacitación al "desarrollo de capacidades y habilidades individuales de los miembros del grupo”. Esta metodología permitió generar un espacio de capacitación, que integra el hacer, el sentir y el pensar, en el cual se desarrollan procesos de construcción y apropiación del índice de satisfacción propuesto. De este modo, se desarrollaron talleres integrados que tuvieron un desenvolvimiento progresivo ya que se fueron desencadenando debido a los intereses y necesidades sentidas de los participantes

\section{RESULTADOS}

\section{Índice de Satisfacción al cliente}

El índice de satisfacción del cliente se obtiene a partir de dos indicadores parciales, que se explicitan a continuación:

1) Índice de satisfacción del cliente respecto de los servicios que brinda el hotel: expresa la satisfacción del cliente con el desempeño de la empresa con respecto a los servicios que brinda.

La tabla número 1 muestra los 22 indicadores evaluados para cada hotel. Cada indicador tiene un puntaje que oscila entre 1 y 5 puntos. La suma de la totalidad de los ítems, de acuerdo al puntaje otorgado a cada ítem, será dividida por el total de respuestas obtenidas en cada ítem para obtener la "respuesta promedio". Luego se obtiene el índice de satisfacción con los servicios, mediante la confección de un índice ponderado teniendo en cuenta la respuesta promedio de cada ítem y la cantidad de respuestas de los mismos 
Tabla $\mathrm{N}^{\circ}$ 1: Indicadores de servicios evaluados para cada Hotel

\begin{tabular}{|c|l|}
\hline No Indicador & Nombre \\
\hline $1 .-$ & Apariencia externa del hotel \\
\hline $2 .-$ & Decoración, diseño y señalización del interior \\
\hline $3 .-$ & Localización del hotel \\
\hline $4 .-$ & Facilidad de acceso al hotel \\
\hline $5 .-$ & Desayuno \\
\hline $6 .-$ & Limpieza del hotel \\
\hline 7.- & Amplitud de los baños \\
\hline $8 .-$ & Amplitud de las habitaciones \\
\hline $9 .-$ & Tv por cable en las habitaciones \\
\hline $10 .-$ & Comodidad de las camas \\
\hline $11 .-$ & Higiene y conservación de las toallas y sábanas \\
\hline $12 .-$ & Luminosidad de las habitaciones \\
\hline $13 .-$ & Accesorios para higiene personal del huésped en los baños \\
\hline $14 .-$ & Calefacción/aire acondicionado en las habitaciones \\
\hline $15 .-$ & Vista exterior desde las habitaciones \\
\hline $16 .-$ & Rapidez en la atención \\
\hline $17 .-$ & Apariencia del personal \\
\hline $18 .-$ & Atención personalizada \\
\hline $19 .-$ & Personal con voluntad para resolver problemas a los pasajeros \\
\hline $20 .-$ & Información precisa y veraz otorgada al cliente \\
\hline $21 .-$ & Seguridad para el pasajero y sus pertenencias \\
\hline $22 .-$ & Personal con conocimiento de idioma extranjeros \\
\hline
\end{tabular}

Elaboración propia

2) Índice de satisfacción del cliente respecto del precio en relación a la calidad percibida: expresa la satisfacción del cliente con la relación precio pagado/calidad del servicio recibido

Seguidamente se obtiene un indicador único de la satisfacción del cliente, calculado sobre la base del índice 1 y el índice 2, ponderados de acuerdo a la importancia 
otorgada por los clientes al servicio y la calidad. La ponderación la establecen lo propios entrevistados.

Este indicador oscila entre 1 y 5 puntos, siendo 1 la satisfacción total y 5 la insatisfacción total.

Se obtendrá un índice de satisfacción para cada hotel a través del promedio de los subíndices obtenidos para el respectivo hotel. Luego de elaborados los índices de satisfacción de cada uno de los hoteles, se podrá conseguir un índice general para los hoteles de la ciudad de Neuquén.

\section{3) Indice precio calidad:}

Representa la satisfacción del cliente respecto de la relación precio pagado/ calidad del servicio percibida. La suma de la totalidad de las respuestas, de acuerdo al puntaje otorgado a cada ítem, será dividida por el total de respuestas obtenidas en esta pregunta para obtener la "respuesta promedio". Este resultado constituye el índice precio/ calidad

\section{4) Índice de satisfacción del cliente para cada hotel:}

Se obtiene a partir de la confección de un promedio con los subíndices calculados en 1) y 2), ponderados según la valoración dada por los clientes del hotel al precio y a la calidad.

Satisfacción del cliente con respecto a cada uno de los hoteles.

HOTELA

\begin{tabular}{|l|c|}
\hline Subindice Servicios & 1,79 \\
\hline Subindice Precio & 2,59 \\
\hline Pond. Serv. & 0,58 \\
\hline Pond. Precio & 0,41 \\
\hline $\begin{array}{l}\text { Índice Satisfacción del } \\
\text { Cliente }\end{array}$ & $\mathbf{2 , 1 2}$ \\
\hline
\end{tabular}

\section{HOTEL B ***}

\begin{tabular}{|l|c|}
\hline Subindice Servicios & 1,83 \\
\hline Subindice Precio & 2,65 \\
\hline Pond. Serv. & 0,57 \\
\hline Pond. Precio & 0,42 \\
\hline $\begin{array}{l}\text { Índice Satisfacción del } \\
\text { Cliente }\end{array}$ & 2,18 \\
\hline
\end{tabular}




\section{HOTEL C ***}

\begin{tabular}{|l|c|}
\hline Subindice Servicios & 2,42 \\
\hline Subindice Precio & 3,18 \\
\hline Pond. Serv. & 0,57 \\
\hline Pond. Precio & 0,42 \\
\hline $\begin{array}{l}\text { Índice Satisfacción del } \\
\text { Cliente }\end{array}$ & $\mathbf{2 , 7 4}$ \\
\hline
\end{tabular}

HOTEL $D$ ***

\begin{tabular}{|l|c|}
\hline Subindice Servicios & 1,54 \\
\hline Subindice Precio & 2,33 \\
\hline Pond. Serv. & 0,58 \\
\hline Pond. Precio & 0,41 \\
\hline $\begin{array}{l}\text { Índice Satisfacción del } \\
\text { Cliente }\end{array}$ & $\mathbf{1 , 8 7}$ \\
\hline
\end{tabular}

\section{HOTEL $E$ ***}

\begin{tabular}{|l|c|}
\hline Subindice Servicios & 1,79 \\
\hline Subindice Precio & 2,83 \\
\hline Pond. Serv. & 0,59 \\
\hline Pond. Precio & 0,41 \\
\hline $\begin{array}{l}\text { Índice Satisfacción del } \\
\text { Cliente }\end{array}$ & $\mathbf{2 , 2 2}$ \\
\hline
\end{tabular}

\section{HOSTAL F ***}

\begin{tabular}{|l|c|}
\hline Subindice Servicios & 1,92 \\
\hline Subindice Precio & 2,70 \\
\hline Pond. Serv. & 0,60 \\
\hline Pond. Precio & 0,39 \\
\hline $\begin{array}{l}\text { Índice Satisfacción del } \\
\text { Cliente }\end{array}$ & $\mathbf{2 , 2 2}$ \\
\hline
\end{tabular}

\section{HOTEL G ***}

\begin{tabular}{|l|l|}
\hline Subindice Servicios & 1,73 \\
\hline Subindice Precio & 2,42 \\
\hline Pond. Serv. & 0,62 \\
\hline Pond. Precio & 0,37 \\
\hline
\end{tabular}




\begin{tabular}{|l|c|}
\hline $\begin{array}{l}\text { Índice Satisfacción del } \\
\text { Cliente }\end{array}$ & 1,98 \\
\hline
\end{tabular}

HOTEL $\mathrm{H}$ **

\begin{tabular}{|l|c|}
\hline Subindice Servicios & 1,92 \\
\hline Subindice Precio & 2,56 \\
\hline Pond. Serv. & 0,55 \\
\hline Pond. Precio & 0,45 \\
\hline $\begin{array}{l}\text { Índice Satisfacción del } \\
\text { Cliente }\end{array}$ & $\mathbf{2 , 2 1}$ \\
\hline
\end{tabular}

HOTEL I **

\begin{tabular}{|l|c|}
\hline Subindice Servicios & 1,83 \\
\hline Subindice Precio & 2,54 \\
\hline Pond. Serv. & 0,49 \\
\hline Pond. Precio & 0,50 \\
\hline $\begin{array}{l}\text { Índice Satisfacción del } \\
\text { Cliente }\end{array}$ & $\mathbf{2 , 1 9}$ \\
\hline
\end{tabular}

\section{HOTEL $\mathrm{H}^{* *}$}

\begin{tabular}{|l|c|}
\hline Subindice Servicios & 1,93 \\
\hline Subindice Precio & 2,53 \\
\hline Pond. Serv. & 0,59 \\
\hline Pond. Precio & 0,67 \\
\hline $\begin{array}{l}\text { Índice Satisfacción del } \\
\text { Cliente }\end{array}$ & $\mathbf{2 , 8 6}$ \\
\hline
\end{tabular}

INDICE GENERAL 2,25 
En función de los diferentes tipos de niveles de satisfacción, se propone la siguiente clasificación por tipo de cliente

\begin{tabular}{|l|c|l|}
\hline \multicolumn{1}{|c|}{ Tipo de Cliente } & Puntaje & \multicolumn{1}{|c|}{ Características } \\
\hline $\begin{array}{l}\text { FIRMES } \\
\text { (Muy Satisfecho) }\end{array}$ & 1 & $\begin{array}{l}\text { Son aquellos clientes que están muy conformes } \\
\text { con los servicios y productos recibidos, siendo } \\
\text { totalmente fieles. }\end{array}$ \\
\hline $\begin{array}{l}\text { FAVORABLES } \\
\text { (Satisfecho) }\end{array}$ & $\begin{array}{c}\text { Mayor a 1 } \\
\text { y } \\
\text { menor a 2 } \\
\text { Son los clientes satisfechos con los servicios y } \\
\text { productos de la empresa, conformes en general y que } \\
\text { pueden ser considerados como 'fieles'. Sin embargo } \\
\text { son clientes que están abiertos a considerar otros } \\
\text { proveedores en la medida que estos los convenzan } \\
\text { de brindarles un mejor servicio }\end{array}$ \\
\hline $\begin{array}{l}\text { VULNERABLES } \\
\text { Indiferente) }\end{array}$ & $\begin{array}{c}\text { Mayor a 2 } \\
\text { yenor a 3 }\end{array}$ & $\begin{array}{l}\text { Son clientes que no están satisfechos totalmente, } \\
\text { encontrando aspectos del servicio que no los } \\
\text { conforman. No son fieles, estando totalmente } \\
\text { abiertos a conocer otros prestadores. }\end{array}$ \\
\hline $\begin{array}{l}\text { INSATISFECHOS } \\
\text { Insatisfecho) }\end{array}$ & $\begin{array}{c}\text { Mayor a 3 } \\
\text { menor a 4 }\end{array}$ & $\begin{array}{l}\text { Son clientes que están insatisfechos en general con } \\
\text { los servicios de la empresa, en aspectos básicos y } \\
\text { estarían buscando cambiar. }\end{array}$ \\
\hline $\begin{array}{l}\text { ALTO RIESGO } \\
\text { (Muy insatisfecho) }\end{array}$ & $\begin{array}{c}\text { Mayor a 4 } \\
\text { menor a 5 }\end{array}$ & $\begin{array}{l}\text { Son los clientes totalmente insatisfechos en } \\
\text { los aspectos básicos del servicio y que estarían } \\
\text { buscando activamente otro prestador para cambiar } \\
\text { sin demoras. }\end{array}$ \\
\hline
\end{tabular}

De acuerdo al indice general obtenido, puede afirmarse que la hotelería de dos, tres y cuatro estrellas de Neuquén capital, posee un tipo de clientela vulnerable presentando las características que se describen en la tabla más arriba propuesta. 


\section{CONCLUSIONES}

Es evidente que el sector está en pleno proceso de consolidación. Para satisfacer las inquietudes de una demanda cada vez más exigente y atender los aspectos fundamentales de cada establecimiento hotelero de la ciudad de Neuquén, es necesario contar con un índice que posibilite medir la satisfacción de los clientes.

Para desarrollarse exitosamente, los hoteles requieren una sólida estructura organizacional donde se prioricen como factor diferencial la calidad en el servicio. Respecto a ello, los establecimientos hoteleros de la ciudad de Neuquén albergan clientes que son "indiferentes" respecto al servicio que los mismos ofrecen, perteneciendo a la categoría vulnerables, clientes que no están satisfechos totalmente, encontrando aspectos del servicio que no los conforman. No son fieles, estando totalmente abiertos a conocer otros proveedores.

En tanto, las diferencias en las formas de aplicación de la gestión de la calidad se hayan relacionadas, en algunos casos con la forma de organización de los establecimientos hoteleros (en algunos casos relacionada con la categoría del hotel, por el tamaño del mismo en cuanto a plazas ofrecidas y cantidad de personal). Los hoteles que pertenecen a las categorías más elevadas se preocupan más por desarrollar una política más bien estructurada y sistemática en cuanto a la gestión de la calidad, mientras que, los hoteles más pequeños prefieren hacerlo de manera más intuitiva y desestructurada, quizá porque los gerentes, dueños o responsables piensen que satisfacer a sus clientes resulte más fácil, ya que ellos saben qué servicios pueden demandar en categorías más bajas.

En cuanto a los resultados obtenidos en relación a la gestión de sus responsables para medir la satisfacción de sus huéspedes, la mayoría de los empresarios hoteleros buscan fidelizar a sus clientes para poder sobrevivir en el mercado.

Invertir en calidad no implica grandes sumas de dinero, la inversión puede ser de tiempo. Cuando se habla de estudiar las características de la demanda no necesariamente se trata de contratar una consultora o realizar una exhaustiva investigación de mercados. En muchos casos con comprender las señales que da el mercado, estar atentos a los cambios en los gustos y preferencias de los huéspedes, observar los comportamientos de los visitantes, etc. Se estará avanzando mucho en lo que se refiere a brindar un servicio de calidad. 


\section{LIMITACIONES}

La ausencia de medición e implementación de I.S.U impide realizar ajustes y mejoras en el diseño y producción de la prestación, lo cual impediría la aplicación de acciones de recuperación de clientes en los casos de que sean necesarios. Las ausencias de mediciones hacen que ciertos aspectos de desempeño no se hagan visibles para los responsables y para el resto de la organización. Asimismo el mejor desempeño económicofinanciero de una empresa y su nivel de participación en el mercado, lleva la necesidad de contar con información confiable y especialmente utilizable

Por otra parte de no mediar intervención alguna en cuanto al uso de herramientas como el propuesto difícilmente las políticas de desarrollo turísticas en general y en especial para el sector contarán con la información necesarias que requieren para llevar adelante el fortalecimiento de la gestión de la Calidad de empresas y destinos para fortalecer la marca Argentina de Calidad la marca Neuquén, Patagonia, etc.

En cuanto al empresario, el no medir desempeño como el I.S.U, provocará mayor desventaja frente a la posibilidad de certificar normas de calidad en el futuro inmediato. 


\section{BIBLIOGRAFIA}

- Bajouth, C (2002) Atributos de compra de los hombres de negocios para el mercado hotelero de la ciudad de Neuquén. Tesina de Grado, Facultad de Turismo, Universidad del Comahue, Neuquén, Argentina.

- Díaz de Santos (1997) La gestión de la calidad. Guías de Gestión de la Pequeña Empresa"

- Dirección Municipal de Turismo de Neuquén (2007) Plan Turístico de la ciudad de Neuquén 2003-2007, Neuquén, Argentina.

- Gazzera Alejandra. Trabajos Prácticos de salida de campo Asignatura Administración de Organizaciones Turísticas I. Universidad del Comahue, Facultad de Turismo, Neuquén, Argentina.

- Gobierno Provincial de Neuquén. Plan Maestro de Turismo para la Provincia el Neuquén 2003-2007

- Hoffman K.D y Bateson J.E. Fundamentos de Marketing de Servicios. Conceptos, estrategias y casos. 2da Edición-ED Thompson, México

- IRAM 30400 (2004) Guía para la interpretación de las Normas ISO 9001:2000 en servicios turísticos

- L.Tamagni et al (2003) Gestión de la Calidad en empresas de servicios turísticos 2001-2003" Proyecto de Investigación, Universidad del Comahue, Facultad de Turismo, Neuquén, Argentina.

- Maestro P; Gallego P; Requejo L; Benito O. ( ) Una propuesta general de análisis de la calidad y la satisfacción en el turismo rural español. Departamento de Administración y Economía de la Empresa-Universidad de Salamanca, España

- Secretaría de Turismo de la Nación. Plan Federal Estratégico de Turismo Sostenible 2016. Argentina.

- Wonham D. (2004) Satisfacción de las necesidades de las Pymes turísticas-recreativas locales de la ciudad de Neuquén a través de las políticas del estado Municipal. Tesina de Grado. Facultad de Turismo, Universidad del Comahue, Argentina.

- Whiteley Richard (1994) La empresa consagrada al cliente. Cómo ser mejor que la competencia. Buenos Aires, Argentina

Recibido: 25/09/2008

Aprobado: 19/11/2008

Arbitrado Anónimamente. 